\title{
Isolation and Identification of Some Chemical Constituents and Antimicrobial Activity of Two Lamiaceae Plants Growing in Saini
}

\author{
K. A. Abdelshafeek ${ }^{\#}$, H. A. Elgendy ${ }^{*}$, M. M. EIMissiry and \\ M. M. Seif EINasr \\ Phytochemistry Dept., National Research Center and \\ *Pharmacog. Dept., Fac. of Pharmacy, Misr Univ. for Sci. and \\ Tech. (MUST), 6- October City, Giza, Egypt.
}

\begin{abstract}
7 WO plants known as Nepeta septemcrenata (N.S.) and Otostegia fruticosa (O.F.) of family Lamiaceae were chosen for the present study. The volatile oils (V. oil) of aerial parts of both plants were isolated by hydrodistillation method and their constituents were identified using GC/MS analysis. It was found that nepetalactone $(53.65 \%)$ and caryophylline oxide $(60.86 \%)$ are the main components in both N.S. and O.F., respectively. The lipid constituents were extracted with pet. ether and fractionated to fatty alcohols, unsaponifiable materials and fatty acids which were identified by GLC analyses. The antimicrobial activity of different extracts of both plants (V. oil, pet. Ether, Fatty alc., Fatty acids (F. acids), 70\% methanol, Chloroform and Ethyl acetate)were evaluated using disc diffusion method against gram positive and gram negative bacteria in addition to fungi. The results proved that, the V. oil and ethyl acetate extract of N.S. are the most effective on E. coli, while, the F. acids fraction and $70 \%$ alc. extract of O.F. exhibited the highest activity against Staphylococcus aurous and Asparaglus niger, respectively.
\end{abstract}

Keywords: Lamiaceae, Nepeta septemcrenata, Otostegia fruticosa, Volatile oil, Lipid constituents and Antimicrobial activity.

Many of the species belonging to Lamiaceae family are aromatic and have been used as flavoring agents, spices and in the manufacture of perfumes. Two plants (Nepeta septemcrenata Ehrenb. [N.S.] and Otostegia fruticosa Forssk [O.F.]) from this family were chosen for investigation. The previous phytochemical studies of this family proved the isolation of many of essential oils, flavonoids, iridoids and phenolic acids ${ }^{(1)}$. The Nepeta genus is represented in Egypt by only one endemic species known as $N$. septemcrenata. It is growing in South Saini, named as Elghomissa in Arabic and used by the native Bedouins as antipyretic, sedative and in sore throat. While, Otostegia fruticosais the most common species of Otostegia genus ${ }^{(2-4)}$. Zuhal et al. ${ }^{(5)}$ isolated two iridoid glycosides, ixoroside and nepetanudoside B ; one phenylpropanoid glycoside, coniferine; two flavone glycosides, apigenin 7-O-glucuronide and apigenin 7-O-glucopyranoside; triterpenes, oleanolic acid and ursolic acid; and sterol, $\beta$-sitosterol from the aerial parts of Nepeta heliotropifolia while, Khan et $a .^{(6)}$ isolated a tetracyclic

\#Corresponding author: mobile phone: 00201155470568

Email: khabdelhady@gmail.com 
triterpenediol (Nepetadiol), in addition, lawsonin and lawsonic acid from the chloroform-soluble portion of Nepeta suavis.

Ashraf et $a l .{ }^{(7)}$ isolated an isopimarane-type diterpene identified as:1ahydroxy-7a,14a,18-triacetoxy-isopimara-8,15-diene from the ethanol extract of the aerial parts of $N$. septemcrenata.

Tauheeda et al ${ }^{(8)}$ investigated the essential oils of $N$. laevigata and N. elliptica and identified the main constituents as: $\alpha$-Citronellol, germacrene $\mathrm{D}, \alpha$-caryophyllene , $\beta$-bisabolol oxide B, $\beta$-bourbonene, $\alpha$-humulene, spathulenol and $\beta$ bisabolol were the major ones. Other constituents such as $4 \mathrm{a} \alpha, 7 \alpha, 7 \mathrm{a} \alpha-$ nepetalactone, allo-aromadendrene, and caryophyllene oxide were present in small amounts. In addition, some other constituents such as $\beta$-pinene, 1,8 -cineole, linalool, geraniol, citronellyl acetate, ... etc. were present in trace amounts.

Fariba et $a l .^{(9)}$ studied the volatile constituents and their biological activity from the flowers and fruits of $O$. persica, they identified the main compounds as a-pinene,1-octene-3-Ol, cubenol (from the flowers), whereas diisooctyl phthalate and hexadecanoic acid (from the fruits). The antioxidant of flowers oil is greater than the fruits oil. Four trans-clerodane diterpenoids provisionally named as limbatolide D, E, F and $\mathrm{G}$ were isolated from the chloroform extract of $O$. limbata $^{(10,11)}$, while otostegindiol ${ }^{(12)}$ which displayed a significant antimalarial activity at doses of 25,50 and $100 \mathrm{mg} / \mathrm{kg}$.

The observed antioxidant activity of $O$. aucheri methanolic extract (OAME) was significant and it was compared with the standard BHT inhibition method. The $\mathrm{IC}_{50}$ value obtained of methanolic extract was $2.23 \mu \mathrm{g} / \mathrm{ml}$. The results obtained from the oral administration of OAME $(1.25 \mathrm{~g} / \mathrm{kg})$ proved no hypoglycemic effect in normal and type I diabetic rats. However, the OAME significantly lowered serum glucose level in type II diabetic models ${ }^{(13)}$.

Anwar et $a l .^{(14)}$ examined the antimicrobial effect of O.limbata extracts obtained with solvent of different polarities (ethanol, dimethylsulphoxide (DMSO) and methanol) on six different bacteria. The results values showed that S.aureus was the most sensitive against all three extracts, where, the highest zone of inhibition value against DMSO extract was $19 \mathrm{~mm}$. No antimicrobial activity was observed against the $P$. aeruginosa, while only ethanolic extract showed some effect against $E$. coli with value of $11.5 \mathrm{~mm}$.

Mohammad et al. ${ }^{(15)}$ proved that $O$. persica extract pretreatment significantly protected the renal injury from skeletal muscle ischemia-reperfusion. The aim of this study was to isolate V.oils, some lipid constituents and evaluates the antimicrobial effect of different extracts of both $N$. septemcrenata [N.S.] and $O$. fruticosa [O.F.]). 
Materials and Methods

\section{Plant material}

Both N.S. and O.F. herbs were collected from St. Catherine region (Southern Sinai), both plants were collected in May during the flowering stage, dried in shade and grinded to fine powder. The plants were kindly, identified by prof. Dr. Ibrahim Algarf, Botany Dept., Faculty of Sci., Cairo Univ., and the voucher specimens were deposited at NRC herbarium.

\section{Extraction of the volatile oils}

About 250g of both fresh aerial parts of both plants (N.S. and O. F.) were subjected to hydrodistillation method for about three hours according to Gunther method $^{(16)}$. The obtained oil was removed, separately, after complete distillation and dried over anhydrous sodium sulphate to give a pale yellow oil having a characteristic odor for each plant (for N.S.0. $26 \%$ and $0.08 \%$ for O.F. v/w, respectively) and kept in refrigerator to GC/MS analysis.

\section{GC/MS analysis of $V$. oils of both plants}

The V. oils were subjected to GC/MS analysis using the following conditions: instrument, Hewlett PackerdHP 5890 gas chromatograph series II with electronic pressure control equipped with an HP5972mass selective detector. Column: HP$5 \mathrm{MS}$ capillary column (30m, $0.25 \mathrm{~mm}$ i.d., $0.25 \square \mathrm{m}$ film). Temp.: oven: $38^{\circ} \mathrm{C}$ up to $250^{\circ} \mathrm{C}, 3^{\circ} \mathrm{C} / \mathrm{min}$., injector: $50^{\circ} \mathrm{C}$, injection splitless. The mass spectra were measured in EI mode scan at 70 ev from 30-550 mass unit, using different data bank of NIST and Wiley $275 \mathrm{~L}$ libraries for the identification of the V. oil components according to their GC retention time and matching with as well as by comparison of the with fragmentation patterns of their mass spectra with those reported in the literature ${ }^{(17,18)}$ as shown in Tables $1 \& 2$.

\section{Extraction of lipid constituents}

About $1.0 \mathrm{Kg}$ of the dried powder of both plants were extracted with petroleum ether (b.r. $40-60{ }^{\circ} \mathrm{C}$ ) in a Soxhlet apparatus. The pet. ether extracts were passed through fuller's earth, filtered, dried over anhydrous sodium sulphate and evaporated in vacuo at $40{ }^{\circ} \mathrm{C}$ till dryness to give a pale yellow residues $(9.60 \mathrm{~g}$ and $4.30 \mathrm{~g})$ for N.S. and O.F., respectively. These residues were dissolved in boiling acetone $(250 \mathrm{ml})$ and left overnight at room temperature. Amorphous precipitates were filtered, washed with cold acetone and recrystallized from chloroform/methanol to give bright white crystals $(2.10 \mathrm{~g})$ and $(0.95 \mathrm{~g})$ of acetone insoluble fraction (Fatty Alcohols mixture). The filtrates (Acetone soluble fraction) were evaporated till dryness $(7.1 \mathrm{~g})$ and $(3.0 \mathrm{~g})$ and subjected to saponification process ${ }^{(1)}$ to afford the unsaponifiable materials, fatty acid methyl esters of both plants .

GC/MS analysis of fatty alcohols of both plants

The fatty alcohols were analyzed using the following conditions, instrument, MS Model 88 SW/HW rev.. Column: DB-1 (crosslinked methyl silicone gum, 
$3 \mathrm{~m}, 0.2 \mathrm{~mm}$ i.d., $0.33 \mu \mathrm{m}$ film). Temp.: source: $200^{\circ} \mathrm{C}$, analyzer $220^{\circ} \mathrm{C}$, carrier gas: helium $600 \mathrm{cc} / \mathrm{min}$., injection splitless. The results were summarized in Table 3.

GLC analysis of unsaponifiable matters and fatty acid methyl esters

The GLC analyses were carried out using the following conditions; Instrument: Varian model 3700GC. Column for unsap. : 10\% OV-101 on chromsorb W/HP, 80/100, ( $2 \mathrm{~m}$ stainless steel, $0.25 \mathrm{~mm}$ i.d.), Column for fatty acid methyl esters:15\% DEGS on chromsorb bW/AW, 80/100, ( $2 \mathrm{~m}$ stainless steel, $0.25 \mathrm{~mm}$ i.d.), Temp. for unsap:column: $70^{\circ} \mathrm{C}$ up to $270^{\circ} \mathrm{C}, 4^{\circ} \mathrm{C} / \mathrm{min}$, injector: $280^{\circ} \mathrm{C}$., Detector (FID): $290^{\circ} \mathrm{C}$.Temp. for fatty acid methyl esters: column: $70^{\circ} \mathrm{C}$ up to $190^{\circ} \mathrm{C}, 4^{\circ} \mathrm{C} / \mathrm{min}$, injector: $240^{\circ} \mathrm{C}$., Detector : $280^{\circ} \mathrm{C}$, Flow Rates for both of them: $\mathrm{N}_{2}$ and $\mathrm{H}_{2}: 30 \mathrm{ml} / \mathrm{min}$, Air: $300 \mathrm{ml} / \mathrm{min}$ the data were tabulated in Tables $4 \& 5$.

Antimicrobial activity study

The study of the antimicrobial activity of different extracts of both plants (V. oil, pet. ether, Fatty alcohols, F. acids, unsap. Fraction, $70 \%$ aqueous methanol, chloroform and ethyl acetate) was carried out using Disc-diffusion method ${ }^{(19)}$ against some selected microorganisms and measuring the inhibition zone in $\mathrm{mm}$ after $24 \mathrm{hr}$ for bacteria and $48 \mathrm{hr}$ for fungi as shown in Table 6.

\section{Tested microorganism}

Cultures of the following organisms were used: St. aureus (ATCC 25923), B. subtillis (ATCC6633), E. coli (ATCC 15036), by microbial Nat. Prod. Dept., NRC. The organisms were supplied through the unit of the Chemistry of Natural and Microbial Products Dept., and the members of this unit isolated the fungi (A. niger and S. cerevisiae), National Research Center, Cairo, Egypt. The control used as antimicrobial are Tetracycline as antibacterial and Metronidazole as antifungal. The microbiological media are Nutrient broth and Nutrient agar (DIFCO laboratories, Detroit, Michigan, USA) was used.

\section{Results and Discussion}

The results of GC/MS of the V. oil of N.S. in Table 1 proved that, it is a mixture of 56 compounds in which 47 of them were identified representing 93.23\%. The identified compounds belong to several chemical classes : hydrocarbons (3.64\%), alcohols $(3.98 \%)$, ketones $(5.85 \%)$, acids $(0.07 \%)$, oxides $(0.18 \%)$, esters $(1.83 \%)$, cyano compounds $(2.01 \%)$, etherials $(2.96 \%)$, chlorinated compounds (8.84\%), terpenes (0.15), in addition to the main compound nepetalactone $(53.65 \%)$. It was found that, the characteristic odor of the plant may be due to the presence of this compound; these results are in a good agreement with many investigators ${ }^{(20,21)}$, where they reported that, nepetalactone is considered as a main compound in other Nepeta species. 
TABLE 1. GC/MS data of V. oil of N.S.

\begin{tabular}{|c|c|c|c|c|c|c|}
\hline \multirow{2}{*}{$\begin{array}{l}\text { Peak } \\
\text { no. }\end{array}$} & \multirow{2}{*}{$\begin{array}{c}\mathbf{R}_{\mathrm{t}} \\
(\mathbf{m i n} .)\end{array}$} & \multirow{2}{*}{$\%$} & \multicolumn{3}{|r|}{ Mass data } & \multirow{2}{*}{ Compounds } \\
\hline & & & MW & B.P. & Fragments m/z (\%) & \\
\hline 1 & 3.89 & 8.77 & 106 & 41 & $55(89), 77(84), 91(15)$ & 2-chloro-2-methyl-butane \\
\hline 2 & 7.64 & 0.11 & 86 & 71 & $41(46), 55(11), 86(50)$ & -1methoxy1-butene \\
\hline 3 & 8.16 & 0.07 & 140 & 77 & $41(50), 69(24), 89(14)$ & 2.3-dichloro-2-methyl-butane \\
\hline 4 & 9.41 & 0.23 & 98 & 69 & $42(59), 55(85), 98(79)$ & 3-methylcyclopentanone \\
\hline 5 & 15.60 & 0.09 & 143 & 93 & $57(39), 71(13), 107(38)$ & Unknown \\
\hline 6 & 16.22 & 0.36 & 128 & 57 & 27(30), 55(32),72(15) & 4-Ol-7-octene \\
\hline 7 & 16.57 & 0.26 & 128 & 43 & $57(80), 71(52), 99(47)$ & 3-octanone \\
\hline 8 & 16.97 & 0.14 & 126 & 69 & $41(61), 56(68), 98(47)$ & 2,6-dimethyl cyclohexanone \\
\hline 9 & 18.33 & 0.66 & 134 & 119 & $82(21), 91(34), 134(22)$ & $P$-cymene \\
\hline 10 & 18.66 & 0.79 & 124 & 81 & 43(68), 77(9), 109(83) & 1-acetyl-1-cyclohexene \\
\hline 11 & 19.54 & 0.16 & 124 & 124 & $55(22), 81(90), 96(48)$ & Bicycli[3.3.1] nonane \\
\hline 12 & 19.74 & 0.74 & 138 & 69 & $41(22), 81(90), 96(48)$ & Comphenilone \\
\hline 13 & 20.07 & 0.17 & 136 & 69 & $55(56), 83(20), 97(42)$ & Unknown \\
\hline 14 & 20.94 & 2.20 & 170 & 59 & $43(65), 68(27), 111(22)$ & Cis-linalool oxide \\
\hline 15 & 21.74 & 1.38 & 170 & 59 & $43(65), 68(27), 111(22)$ & trans-linalool oxide \\
\hline 16 & 22.59 & 0.13 & 124 & 109 & $43(53), 81(55), 124(09)$ & 6-methyl-3,5-heptadien-2-one \\
\hline 17 & 22.76 & 0.07 & 172 & 55 & 67(70), 93(65),111(74) & $\begin{array}{l}\text { Furan-2(2-ethoxy-1- } \\
\text { methoxyeth) }\end{array}$ \\
\hline 18 & 23.08 & 0.66 & 155 & 99 & 54(74),67(43), 128(23) & 1-octen-1-Ol acetate \\
\hline 19 & 24.54 & 0.06 & 138 & 81 & $68(48), 109(24), 138(40)$ & 1.2-dihydrolimonene \\
\hline 20 & 25.69 & 0.17 & 170 & 68 & 59(73), 94(50), 155(9) & $\begin{array}{l}\text { 2-H-pyran-3-OL6-ethenyl- } \\
\text { tetrahydro-2,2,6-trimethyl }\end{array}$ \\
\hline 21 & 25.98 & 0.22 & 170 & 68 & $43(32), 59(65), 94(51)$ & Unknown \\
\hline 22 & 27.96 & 2.81 & 138 & 138 & $55(23), 67(18), 123(98)$ & $p$-methoxyanisol \\
\hline 23 & 28.21 & 0.42 & 138 & 67 & $41(30), 82(38), 95(15)$ & 1-pentyl cyclopentene \\
\hline 24 & 28.78 & 0.28 & 184 & 57 & 67(83), 82(93), 100(34) & $\begin{array}{l}\text { Cis-3-hexenyl-2-methyl- } \\
\text { butanoate }\end{array}$ \\
\hline 25 & 28.97 & 0.23 & 184 & 82 & 67(84), 57(59),41(52) & $\begin{array}{l}\text { Cis-3-hexenyl-3-methyl- } \\
\text { butanoate }\end{array}$ \\
\hline 26 & 30.47 & 0.18 & 166 & 81 & 41(89), 67 (91), 95 (34) & 1-dodecyne \\
\hline 27 & 30.69 & 0.18 & 164 & 104 & 51(15), 91(74), 164(24) & Benzene propanoic methyl ester \\
\hline 28 & 31.03 & 0.28 & 152 & 81 & $41(42), 69(56), 100(95)$ & 1-methyl comphenilone \\
\hline 29 & 31.40 & 0.13 & 194 & 159 & 43(32), 69(32), 107(15) & $\begin{array}{l}\text { 2-H-benzopyran-3,4,4a,5,6,8a- } \\
\text { hexa-hydroxy 2,5,5,8a- } \\
\text { tetramethyl }\end{array}$ \\
\hline 30 & 32.08 & 0.38 & 150 & 135 & $91(16), 115(22), 150(30)$ & Thymol \\
\hline 31 & 32.69 & 12.7 & 150 & 135 & 77(19), 91(15), 150(27) & Carvacrol \\
\hline 32 & 32.97 & 0.5 & 150 & 55 & 67(97), $83(29), 135(46)$ & Unknown \\
\hline 33 & 33.21 & 0.36 & 152 & 41 & $27(59), 81(62), 124(31)$ & $\begin{array}{l}\text { cis-bicyclo[5.2.0]nonane-1,7- } \\
\text { dimethyl }\end{array}$ \\
\hline 34 & 35.25 & 53.65 & 168 & 81 & 69(90), 123(85), 166(77) & Nepetalactone \\
\hline 35 & 35.69 & 2.01 & 153 & 153 & $69(13), 81(51), 105(90)$ & naphthlene-2-carbonitrile \\
\hline 36 & 35.89 & 0.88 & 189 & 81 & $55(37), 95(31), 153(78)$ & Unknown \\
\hline 37 & 36.86 & 1.09 & 152 & 82 & $41(33), 67(78), 95(51)$ & $\begin{array}{l}\text { 3,7,7-trimethyl, bicyclo[4.1.0] } \\
\text { heptane-2-one }\end{array}$ \\
\hline 38 & 38.92 & 0.07 & 224 & 69 & $93(41), 121(21), 136(24)$ & $\begin{array}{l}\text { 2-methyl-Propanoic acid-3,7- } \\
\text { dimethyl,2,6-octadiene }\end{array}$ \\
\hline 39 & 39.16 & 0.31 & 204 & 81 & $55(51), 95(41), 180(81)$ & Unknown \\
\hline 40 & 39.40 & 0.09 & 204 & 119 & $41(33), 96(38), 105(53)$ & Thujopsene \\
\hline
\end{tabular}


TABLE 1. Cont.

\begin{tabular}{|c|c|c|c|c|c|c|}
\hline \multirow{2}{*}{$\begin{array}{l}\text { Peak } \\
\text { no. }\end{array}$} & \multirow[b]{2}{*}{$\mathbf{R}_{t}$ (min.) } & \multirow[b]{2}{*}{$\%$} & \multicolumn{3}{|r|}{ Mass data } & \multirow[b]{2}{*}{ Compounds } \\
\hline & & & MW & B.P. & Fragments & \\
\hline 41 & 39.61 & 0.16 & 204 & 105 & $55(68), 67(80), 93(91)$ & Valencene \\
\hline 42 & 40.22 & 0.32 & 204 & 81 & $57(68), 105(80), 153(74)$ & Germacrene-D \\
\hline 43 & 40.71 & 0.22 & 196 & 69 & $57(78), 121(35), 161(15)$ & Geranyl acetate \\
\hline 44 & 40.85 & 0.26 & 154 & 123 & $67(30), 82(72), 136(23)$ & $p$-menth-3-ene-9-Ol \\
\hline 45 & 41.10 & 0.42 & 202 & 159 & $91(5), 105(17), 128(11)$ & Calamenene \\
\hline 46 & 41.88 & 0.47 & 200 & 157 & $81(54), 142(48), 166(15)$ & $\begin{array}{l}\text { 4-ethyl-1,1,6-Trimethyl-1,2- } \\
\text { dihydronaphthalene }\end{array}$ \\
\hline 47 & 42.20 & 1.93 & 138 & 67 & 81(48), 95(63), 109(93) & spiro[4.4]nonan-2-one \\
\hline 48 & 42.62 & 0.11 & 206 & 81 & $55(11), 123(18), 168(14)$ & Dihydro- $\beta$-humulene \\
\hline 49 & 42.74 & 0.46 & 204 & 80 & 53(10), 123(59),161(16) & Unknown \\
\hline 50 & 43.00 & 0.32 & 166 & 105 & 51(53), 67(29), 82(79) & Unknown \\
\hline 51 & 43.82 & 0.16 & 196 & 123 & $51(21), 67(75), 82(84)$ & Ethyl chrysanthemate \\
\hline 52 & 44.39 & 0.26 & 206 & 82 & $41(96), 107(47), 191(15)$ & $\begin{array}{c}\text { 6,10-dimethyl,3-(1-methyl } \\
\text { ethyl)1-cyclodecene }\end{array}$ \\
\hline 53 & 44.92 & 0.16 & 206 & 79 & 55(57), 93(80), $164(33)$ & Unknown \\
\hline 54 & 46.08 & 0.24 & 222 & 181 & 95(94), 151(85),165(17) & Widdrol \\
\hline 55 & 57.38 & 0.05 & 275 & 55 & 81(84), 137(38),257(35) & Manoyloxide \\
\hline 56 & 61.16 & 0.15 & 266 & 209 & $\begin{array}{c}\text { 147(40),171(61), } \\
251(98)\end{array}$ & Equilenin \\
\hline
\end{tabular}

The data of GC/MS of V. oil of O.F. in Table 2 revealed the presence of 13 compounds in which caryophyllin oxide is the major one $(60.86 \%)$. The other compounds include hydrocarbons $(2.8 \%)$, alcohols $(4.36 \%)$, aldehydes $(2.15 \%)$, esters $(4.21 \%)$, and nitrogenous compounds (1.39\%). It was found that the oil content of N.S. is higher than that of O.F. This result is coincided with that reported on Lamiaceae family, where it is divided into two subfamilies Lamioideae of poor oil content and Nepetoideae of rich oil content so, we can consider N.S. is belonging to Nepetoideae while O.F. is belonging to Lamioideae ${ }^{(4)}$.

TABLE 2. GC/MS data of V. oil of O.F.

\begin{tabular}{|c|c|c|c|c|c|c|}
\hline \multirow{2}{*}{$\begin{array}{c}\text { Peak } \\
\text { no. }\end{array}$} & $\begin{array}{c}\mathbf{R}_{\mathbf{t}} \\
(\mathbf{m i n} \text { ) }\end{array}$ & $\boldsymbol{\%}$ & $\mathbf{M W}$ & $\mathbf{B . P .}$ & Fragments m/z(\%) & \multirow{2}{*}{ Compounds } \\
\hline 1 & 4.66 & 0.23 & 119 & 83 & $53(16), 69(20), 104(17)$ & Chloroform \\
\hline 2 & 20.64 & 2.15 & 112 & 57 & $55(51), 83(13), 89(10)$ & 2-heptenal \\
\hline 3 & 21.96 & 1.86 & 152 & 91 & $65(9), 105(14), 119(14)$ & Cis-carveol \\
\hline 4 & 24.22 & 2.5 & 154 & 71 & $55(21), 93(54), 136(17)$ & 4-terpineol \\
\hline 5 & 33.10 & 1.91 & 204 & 119 & $93(49), 105(89), 161(80)$ & $\alpha$-copaene \\
\hline 6 & 33.47 & 4.16 & 204 & 81 & $67(13), 91(15), 161(32)$ & $\beta$-bourbonene \\
\hline 7 & 34.91 & 4.39 & 204 & 93 & $69(80), 119(30), 189(7)$ & $\alpha$-cis-bergamotene \\
\hline 8 & 38.70 & 9.16 & 204 & 69 & $53(14), 93(86), 189(12)$ & $\beta$-bisablene \\
\hline 9 & 38.93 & 2.09 & 204 & 161 & $79(30), 107(60), 122(28)$ & $\alpha$-cadenen \\
\hline 10 & 40.30 & 1.39 & 121 & 106 & $52(11), 78(29), 121(28)$ & Pyridine-2-(1-methyl ethyl) \\
\hline 11 & 41.56 & 60.86 & 220 & 79 & $55(54), 91(78), 187(12)$ & Caryophyllene oxide \\
12 & 42.50 & 5.09 & 138 & 67 & $53(36), 96(69), 123(21)$ & 4-decyne \\
\hline 13 & 44.96 & 4.21 & 198 & 93 & $41(89), 69(50), 136(4)$ & linalyl acetate \\
\hline
\end{tabular}

Egypt. J. Chem. 59, No.1 (2016) 
The data in Table 3 showed that, N.S. contain seven fatty alcohols in which octacosanol and triacontanol are the main $(34.68 \%$ and $27.04 \%$, respectively) while O.F. include five fatty alcohols with triacontanol as main $(47.58 \%)$.

TABLE 3. GC/MS data of fatty alcohols of both N.S. and O.F.

\begin{tabular}{|c|c|c|c|l|c|c|}
\hline \multirow{2}{*}{$\begin{array}{c}\text { Peak } \\
\text { no. }\end{array}$} & \multirow{2}{*}{$\begin{array}{c}\text { Ret. Time } \\
\text { (min.) }\end{array}$} & \multicolumn{2}{|c|}{ Rel. \% } & \multicolumn{1}{|c|}{ Fatty alc. } & $\begin{array}{c}\text { Molecular } \\
\text { formula }\end{array}$ & M. Wt. \\
\hline 1 & 11.18 & 12.20 & - & Hexacosanol & $\mathrm{C}_{26} \mathrm{H}_{54} \mathrm{O}$ & 382 \\
\hline 2 & 12.53 & 4.90 & - & Heptacosanol & $\mathrm{C}_{27} \mathrm{H}_{56} \mathrm{O}$ & 396 \\
\hline 3 & 14.16 & 34.68 & 29.5 & Octacosanol & $\mathrm{C}_{28} \mathrm{H}_{58} \mathrm{O}$ & 410 \\
4 & 15.39 & 7.06 & 4.05 & Nonacosanol & $\mathrm{C}_{29} \mathrm{H}_{60} \mathrm{O}$ & 424 \\
5 & 17.04 & 27.04 & 47.58 & Triacontanol & $\mathrm{C}_{30} \mathrm{H}_{62} \mathrm{O}$ & 438 \\
\hline 6 & 18.24 & 3.89 & 3.31 & Hentriacontanol & $\mathrm{C}_{31} \mathrm{H}_{64} \mathrm{O}$ & 452 \\
\hline 7 & 19.86 & 9.91 & 15.55 & Dotriacontanol & $\mathrm{C}_{32} \mathrm{H}_{66} \mathrm{O}$ & 466 \\
\hline
\end{tabular}

The GLC analysis of the unsap. fraction (Table 4) of both plants revealed the presence of a mixture of n-hydrocarbons, sterols and triterpenes. There is a similarity in the n-hydrocarbon mixtures of both plants but differ only in the percentage of each component. Also, the sterol fraction of N.S. constitutes $8.76 \%$ which contain cholesterol, $\beta$-sitosterol and stigasterol, while that of O.F. represents $9.37 \%$ with campasterol and $\beta$-sitosterol. The triterpene in N.S. is $\alpha$ amyrene $(3.11 \%)$ and in O.F. is $\beta$ - amyrene (4.39\%).

TABLE 4. GLC data of the unsap. fraction of both N.S. and O.F.

\begin{tabular}{|c|c|c|c|c|}
\hline \multirow{2}{*}{ Peak no. } & \multirow{2}{*}{$\begin{array}{l}\text { Ret. Time } \\
\text { (min.) }\end{array}$} & \multicolumn{2}{|c|}{$\%$} & \multirow{2}{*}{ Compounds } \\
\hline & & N.S. & O.F. & \\
\hline 1 & 14.91 & 0.70 & 5.28 & Dodecane, n-C 12 \\
\hline 2 & 19.65 & 0.11 & 0.90 & Tridecane, n-C 13 \\
\hline 3 & 23.02 & 0.68 & 1.70 & Tetradecane, n-C 14 \\
\hline 4 & 25.86 & 0.22 & 1.45 & Pentadecane, n-C 15 \\
\hline 5 & 31.24 & 0.78 & 1.54 & Heptadecane, n-C 17 \\
\hline 6 & 34.99 & 1.38 & 3.14 & Octadecane, n-C 18 \\
\hline 7 & 36.91 & 0.88 & 0.96 & Nonadecane, n-C 19 \\
\hline 8 & 39.58 & 1.68 & 1.50 & Eicosane, n-C 20 \\
\hline 9 & 41.49 & 2.29 & 1.30 & Heneicosane, n-C 21 \\
\hline 10 & 44.31 & 5.70 & 0.28 & Docosane, n-C 22 \\
\hline 11 & 44.36 & 6.31 & 0.01 & Tricosane, n-C 23 \\
\hline 12 & 47.15 & 30.77 & 6.45 & Lupeol \\
\hline 13 & 48.38 & 3.11 & - & $\beta$ - amyrine \\
\hline 14 & 50.18 & 6.08 & 29.28 & Tetracosane, n-C 24 \\
\hline 15 & 52.41 & 5.46 & 0.52 & Hexacosane, n-C 26 \\
\hline 16 & 53.51 & 4.88 & 2.78 & Squalene \\
\hline 17 & 55.0 & 3.21 & 7.09 & Octacosane, n-C 28 \\
\hline 18 & 57.17 & 2.34 & 4.31 & Triacontane, n-C 30 \\
\hline 19 & 66.73 & 0.58 & - & Cholesterol \\
\hline 20 & 71.96 & - & 0.45 & Campasterol \\
\hline 21 & 76.09 & 5.01 & 8.93 & $\beta$-sitosterol \\
\hline 21 & 79.01 & 3.17 & - & Stigmasterol \\
\hline 22 & 83.63 & 0.29 & 4.39 & $\alpha$-amyrine \\
\hline 23 & --- & 14.16 & 18.55 & unidentified compounds \\
\hline
\end{tabular}

Egypt. J. Chem. 59, No.1 (2016) 
TABLE 5. GLC of FAME of both N.S. and O.F.

\begin{tabular}{|c|c|c|c|l|l|}
\hline \multirow{2}{*}{$\begin{array}{c}\text { Peak } \\
\text { no. }\end{array}$} & $\begin{array}{c}\text { Ret. Time } \\
\text { (min.) }\end{array}$ & \multicolumn{2}{|c|}{ Rel. \% } & \multicolumn{1}{|c|}{ Compounds } & \multirow{2}{*}{ Molecular formula } \\
\cline { 3 - 4 } & & N.S. & O.F. & & \\
\hline 1 & 4.17 & - & 0.08 & Butyric acid & $\mathrm{C}_{4} \mathrm{H}_{8} \mathrm{O}_{2}, \mathrm{C} 4(0)$ \\
\hline 2 & 6.67 & - & 0.30 & Caproic acid & $\mathrm{C}_{6} \mathrm{H}_{12} \mathrm{O}_{2}, \mathrm{C} 6(0)$ \\
\hline 3 & 8.37 & - & 0.20 & Caprylic acid & $\mathrm{C}_{8} \mathrm{H}_{16} \mathrm{O}_{2}, \mathrm{C} 8(0)$ \\
\hline 4 & 10.24 & - & 0.40 & Pelargonic acid & $\mathrm{C}_{9} \mathrm{H}_{18} \mathrm{O}_{2}, \mathrm{C} 9(0)$ \\
\hline 5 & 13.16 & - & 0.32 & Capric acid & $\mathrm{C}_{10} \mathrm{H}_{20} \mathrm{O}_{2}, \mathrm{C} 10(0)$ \\
\hline 6 & 16.65 & - & 0.36 & Undecanoic acid & $\mathrm{C}_{11} \mathrm{H}_{22} \mathrm{O}_{2}, \mathrm{C} 11(0)$ \\
\hline 7 & 19.03 & - & 0.35 & Lauric acid & $\mathrm{C}_{12} \mathrm{H}_{24} \mathrm{O}_{2}, \mathrm{C} 12(0)$ \\
\hline 8 & 22.39 & 1.16 & 1.6 & Tridecanoic acid & $\mathrm{C}_{13} \mathrm{H}_{26} \mathrm{O}_{2}, \mathrm{C} 13(0)$ \\
\hline 10 & 25.51 & 2.13 & 0.48 & Myrestic acid & $\mathrm{C}_{14} \mathrm{H}_{28} \mathrm{O}_{2}, \mathrm{C} 14(0)$ \\
\hline 11 & 27.85 & 28.39 & 19.0 & Palmitic acid & $\mathrm{C}_{16} \mathrm{H}_{32} \mathrm{O}_{2}, \mathrm{C} 16(0)$ \\
\hline 12 & 29.80 & 1.42 & 1.04 & Margaric acid & $\mathrm{C}_{17} \mathrm{H}_{34} \mathrm{O}_{2}, \mathrm{C} 17(0)$ \\
\hline 13 & 31.88 & 10.21 & 8.0 & Stearic acid & $\mathrm{C}_{18} \mathrm{H}_{36} \mathrm{O}_{2}, \mathrm{C} 18(0)$ \\
\hline 14 & 32.82 & 12.48 & 5.60 & Oleic acid & $\mathrm{C}_{18} \mathrm{H}_{34} \mathrm{O}_{2}, \mathrm{C} 18(1)$ \\
\hline 15 & 34.65 & 9.87 & 16.0 & Linoleic acid & $\mathrm{C}_{18} \mathrm{H}_{32} \mathrm{O}_{2}, \mathrm{C} 18(2)$ \\
\hline 16 & 35.65 & 8.35 & 3.0 & Linolenic acid & $\mathrm{C}_{18} \mathrm{H}_{30} \mathrm{O}_{2}, \mathrm{C} 18(3)$ \\
\hline 17 & 37.59 & 13.02 & 43.10 & arachidic acid & $\mathrm{C}_{20} \mathrm{H}_{40} \mathrm{O}_{2}, \mathrm{C} 20(0)$ \\
\hline 18 & 39.97 & 12.96 & - & Behenic acid & $\mathrm{C}_{22} \mathrm{H}_{44} \mathrm{O}_{2}, \mathrm{C} 22(0)$ \\
\hline
\end{tabular}

The data in Table 6 proved that, the V. oil of N.S. gave the highest inhibition against E. coli and St. aureus where are inhibition zone (IZ)19 and $18 \mathrm{~mm}$ respectively, whereas, the $\mathrm{V}$. oil of O.F. gave moderate activity against the same two species with IZ of $11,14 \mathrm{~mm}$. The unsap. fraction of both N.S. and O.F. exhibited highest activity A. niger and $S$. cereviseae, respectively, the lowest activity was found against $B$. sabtilis with unsap. fraction of N.S. and chloroform extract of O.F.

TABLE 6. Antimicrobial activity of different extracts of both N.S. and O.F.

\begin{tabular}{|c|c|c|c|c|c|c|}
\hline \multirow{3}{*}{ Plant } & \multirow{3}{*}{ Extracts } & \multicolumn{5}{|c|}{ Inhibition zone diameter (mm) } \\
\hline & & \multicolumn{2}{|c|}{ Gr. +ve bacteria } & \multirow{2}{*}{$\begin{array}{c}\begin{array}{c}\text { Gr. -ve } \\
\text { bacteria }\end{array} \\
E \text {. coli }\end{array}$} & \multirow{2}{*}{$\begin{array}{l}\text { Fungus } \\
\text { A. niger }\end{array}$} & \multirow{2}{*}{$\begin{array}{c}\text { Yeast } \\
\text { S. cereviseae }\end{array}$} \\
\hline & & St. aureus & B. sabtilis & & & \\
\hline \multirow{8}{*}{ 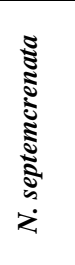 } & Volatile oil & 16 & 8 & 19 & 11 & 14 \\
\hline & Pet. ether & 15 & 8 & 17 & 18 & 18 \\
\hline & Fatty alc. & - & - & - & 10 & - \\
\hline & Fatty acids & 16 & 9 & 14 & 18 & 13 \\
\hline & Unsap. Fr. & - & 8 & 10 & 19 & 9 \\
\hline & $70 \%$ Methanol & 12 & 10 & 17 & 21 & 9 \\
\hline & chloroform & 18 & 10 & 18 & 15 & 12 \\
\hline & Ethyl acetate & 10 & 11 & 22 & 17 & 18 \\
\hline \multirow{8}{*}{$\underbrace{\stackrel{\Xi}{\Xi}}_{0}$} & Volatile oil & 14 & 10 & 11 & 16 & - \\
\hline & Pet. ether & 10 & - & 12 & 16 & - \\
\hline & Fatty alc. & - & - & - & 12 & - \\
\hline & Fatty acids & 16 & 12 & 13 & - & 12 \\
\hline & Unsap. Fr. & - & - & 10 & 13 & 14 \\
\hline & $70 \%$ Methanol & 9 & 10 & 14 & 17 & 11 \\
\hline & chloroform & - & 9 & - & 11 & 10 \\
\hline & Ethyl acetate & 12 & 12 & 11 & 14 & 16 \\
\hline
\end{tabular}

Egypt. J. Chem. 59, No.1 (2016) 
Acknowledgement : The authors are in debt to Prof. Dr. Abdel Hamid A. Hamdy Chemistry of Microbial and Natural Products Dept., National Research Center, Dokki, Giza, Egypt, for providing the different microbial cultures and his help in carrying out of antimicrobial activity of different extracts.

\section{References}

1. Abdelshafeek, K.A., M.Sc. Thesis, Chemistry Dept., Fac. of Sci., Cairo Univ., Egypt (1997).

2. Tackholm, V., Student's Flora of Egypt, Cairo Univ.,2 ${ }^{\text {nd }}$ ed.,coop, Beirut (1974).

3. Barberan, F.A.T., The flavonoid compounds from labiateae. Fitotrapia, 57(2), 6795 (1986).

4. Barberan, F.A.T. and Wollenweber, E., Flavonoid aglycones from the leaf surfaces of some Labiatae species. Pl. Syst. Ecol.173, 109-118 (1990).

5. Zuhal, G.P., Hilal, O., Kuruuzum, Z., Cavit, K.L. and Omur, D., Secondary metabolites from Nepeta heliotropifolia. Turk. J. Chem. 33, 667-675 (2009).

6. Khan, F.U., Hussain, J., Khan, I., Ullah, U. R., Ali, I., Muhammad, Z., Hussain, H. and Shah M. R., Nepetadiol, A new triterpenediol from Nepeta suavis. Chem. Nat. Compds. 47 (2), (2011).

7. Ashraf, T.K., Saharr, G., Ohamedf, M.L., Hamed, F.H. and Markusv, O., A diterpene from N. Septemcrenata. Phytochem. 44 (3), 475-478 (1997).

8. Tauheeda, H., Manzoor, A. R., Abdul, S. S., Khursheed, A. B., Haroon, M. B., Bilal, A. D., Ghulam, H. D. and Mushtaq, A. Q., Chemical composition of the essential oils of $N$. laevigata and $N$. elliptica from India. Chem. Nat. Compds. 47, 3 (2011).

9. Fariba, S., Valiolah, M. and Shirin, M., Comparison of antioxidant and free radical scavenging activities of the essential oils from flowers and fruits of O. persica. Pak. J. Biol. Sci.10 (21), 3895-3899 (2007).

10. Khan, F. and Kousar, H., Two new clerodane diterpenoids from O. limbata. J. Asian Nat. Prods. Res. 9, 91 - 95 (2007).

11. Khan, F., Ahmad, K. , Iqbal, M. and Limbatolide, F., Two new trans-clerodane diterpenoids from O. limbata. Pol. J. Chem. 79 (11), 1757-1762 (2005).

12. Bisrat, D., Animut, A., Bucar, F. and Asres, K., In vivo antimalarial activity of a labdane diterpenoid from the leaves of O. integrifolia. Phytother. Res. 27 (12), 18051809 (2013).

13. Rashid, R., Murtaza, G., Khan, A. K. and Mir, S., Antioxidant and hypoglycemic effect of $O$. aucheri methanolic extract in streptozotocin-induced diabetic male longevans rats. Acta Poloniae Pharm. Drug Res. 71 (4), 631- 635 (2014). 
14. Anwar, N., Salik, S. and Ahmad, D., Antibacterial activity of O. limbata. Inter. J. Agri. Biol. 11(5), 647-650 (2009).

15. Mohammad, A.T., Hamed, A.T., Fariborz, M., Poorya, K. and Hesam, A.A., Effect of $O$. persica extraction on renal injury induced by hindlimb ischemiareperfusion: A rat model. Intern. J. Surgery, 13, 124-130 (2015).

16. Guenther, E., The Production of Essential Oils, p. 87: In: E.Guenther (Ed.). The Essential Oils. Krieger Publ. Co., Malabar, Fla (1972).

17. Adams, R.P., Identification of Essential Oil Components by GC-MS. Allured Publ.Corp., Carl Stream, II (1995).

18. Mclafferty, F.W. and Staffer, D.B., The Eiley NBS Registry of Mass Spectral Data, vol. 1-7, Wiley Int. Public (1989).

19. Hamdi, A., M. Sc. Thesis, Pharmacog. Dept., Faculty of Pharmacy, Cairo Univ., Egypt (1979).

20. Heba, I.A., Essential oil and iridoid glycosides of Nepeta septemcrenata. J. Nat. Prod. 3, 103-111 (2010).

21. Wafaa, H.B.H., Mai, A., Ebtesam, E., Ali, E., Nida, N.F. and Zeinab, I.A.E., Chemical composition and antimicrobial activity of the essential oil of Nepeta deflersiana Growing in Saudi Arabia. J. Appl. Sci. Res.10 (12), 51-56 (2014). 
فصل وتعريف بعض المكونات الكيميائية والفاعلية كمضادات للميكروبات لاثنين من نباتات العائلة الثفوية التى تنمو في سيناء

خالــد عبدالهادى ، حسينى عبدالمحسن الجنـدى"، مصطفى المسيـرى و مدحت سيف النصر عيداء

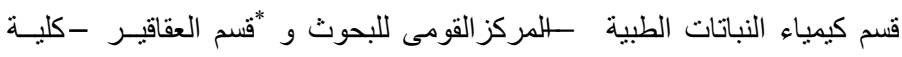

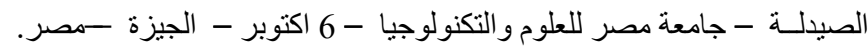

لقد تم اختيار اثنين من النباتات المعروفة باسم نبيتا سبتيمكريناتا (N.S).

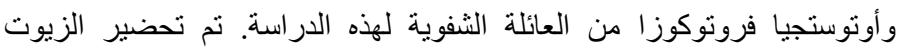

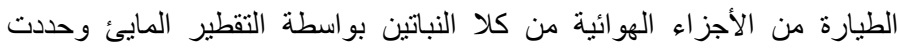

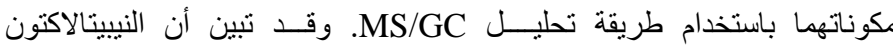

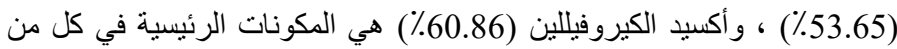

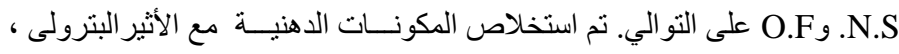

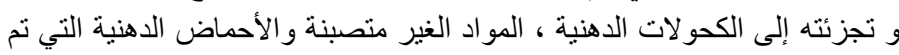

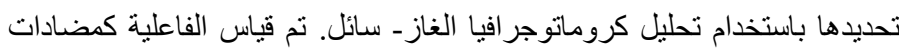

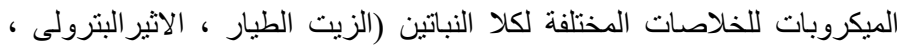

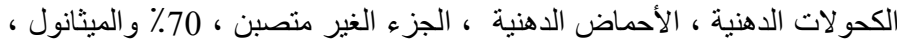

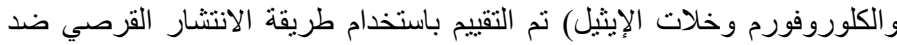
البكتيريا موجبة وسالبة الجر ام بالإضافة إلى الفطريات. 